\title{
FORMATION AND ALIGNMENT OF ELONGATED, FRACTAL-LIKE WATER-ICE GRAINS IN EXTREMELY COLD, WEAKLY IONIZED PLASMA
}

\author{
Kil-Byoung Chai and Paul M. Bellan \\ Applied Physics and Materials Science, California Institute of Technology, 1200 E. California Blvd., \\ Pasadena, CA 91125, USA; kbchai@caltech.edu, pbellan@caltech.edu \\ Received 2014 November 17; accepted 2015 February 6; published 2015 March 30
}

\begin{abstract}
Elongated, fractal-like water-ice grains are observed to form spontaneously when water vapor is injected into a weakly ionized laboratory plasma formed in a background gas cooled to an astrophysically relevant temperature. The water-ice grains form in 1-2 minutes, levitate with regular spacing, and are aligned parallel to the sheath electric field. Water-ice grains formed in plasma where the neutrals and ions have low mass, such as hydrogen and helium, are larger, more elongated, and more fractal-like than water-ice grains formed in plasmas where the neutrals and ions have high mass such as argon and krypton. Typical aspect ratios (length to width ratio) are as great as 5 while typical fractal dimensions are $\sim 1.7$. Water-ice grain lengths in plasmas with low neutral and ion masses can be several hundred microns long. Infrared absorption spectroscopy reveals that the water-ice grains are crystalline and so are similar in constitution to the water-ice grains in protoplanetary disks, Saturn's rings, and mesospheric clouds. The properties and behavior of these laboratory water-ice grains may provide insights into morphology and alignment behavior of water-ice grains in astrophysical dusty plasmas.
\end{abstract}

Key words: dust, extinction - methods: laboratory: solid state - planets and satellites: rings - plasmas protoplanetary disks

Supporting material: animations

\section{INTRODUCTION}

\subsection{Examples of Ice Dusty Plasmas in Astrophysics}

Plasmas consisting of electrons, ions, neutral gas, and dust exist over an enormous range of scales in the universe. Going from large to small scale, examples include the interstellar medium (Zubko et al. 2004), red giant outflows (Jura \& Morris 1985), protoplanetary disks (Pollack et al. 1994; McClure et al. 2012), certain rings of Saturn (Goertz 1989), comet tails (Davies et al. 1997), and terrestrial polar mesospheric clouds (Havnes et al. 1996). Water-ice in these plasmas plays an important role in planetesimal and gas giant formation, disk temperature profile, disk opacity in infrared range, and storage of oxygen (McClure et al. 2012).

Previous discussions of astrophysical dust concentrated on composition rather than plasma physics issues (Pollack et al. 1994; Zubko et al. 2004) although the ionization rate of protoplanetary disks is sufficiently high $\left(10^{-8}-10^{-15}\right)$ to invoke a MHD description (Sano et al. 2000; Ilgner \& Nelson 2006; Wardle 2007). For example, Pollack et al. (1994) presented a detailed model of the composition and radiative properties of dust grains in molecular clouds and accretion disks, but did not discuss how ambient plasma might affect dust properties. Pollack et al. (1994) used observational data to show that the dust particles are made of silicate, metallic iron, water-ice, organics, and troilite and stated that water-ice exists in the form of a mantle on refractory material (i.e., not in the form of a pure ice particle). The abundance of water-ice in protoplanetary disk is as large as silicate and iron (Pollack et al. 1994) while only few per cent of total dust mass in the interstellar medium (Zubko et al. 2004).

Electrical charging of dust grains from interaction with plasma particles causes behavior very different from that of uncharged dust grains. Like-charged dust grains will repel each other and so prevent agglomeration (Praburam \& Goree 1995; Okuzumi 2009;
Matthews et al. 2012). Charged dust grains can produce attractive forces on particles of atomic-scale or larger having either opposite charge or dipole moments; these attractive forces would cause the growth properties of charged dust grains to differ substantially from those of uncharged grains.

Water-ice forms in protoplanetary disks when the ambient temperature is below $170 \mathrm{~K}$ (Hayashi 1981); the radial distance from the star where this temperature occurs is called the snow line. It has been conventionally assumed (e.g., see Kahn 1952; Seki \& Hasegawa 1983) that astrophysical water-ice grains form via heterogeneous rather than homogeneous nucleation, i.e., they form by condensation of water molecules on a nonice, typically silicate, nucleation center. Seki \& Hasegawa (1983) argued that astrophysical ice forms during a cooling process and that because the temperature for heterogeneous nucleation is higher than for homogeneous nucleation, heterogeneous nucleation dominates. However, this argument neglects electrical forces.

Water-ice grains in protoplanetary disks have been modeled as being spherical with radii $a$ in the range 0.01 $\mu \mathrm{m}<a<10 \mathrm{~cm}$ and a power law density distribution $n \sim a^{-p}$ with $p=3.5$ (Miyake \& Nakagawa 1993; McClure et al. 2012). This $p=3.5$ power law results from assuming that grains continuously collide with each other, sometimes coalescing and sometimes fragmenting until an equilibrium density distribution $n \sim a^{-p}$ results (Biermann \& Harwit 1980). On the other hand, monodisperse spherical ice grain models have also been assumed to model ice grains in protoplanetary disks (Oka et al. 2011) and to model formation of ice grains in stellar outflows from mass-losing red giant stars (Jura \& Morris 1985). Meanwhile, some authors proposed that ice grains are nonspherical and elongated (Gupta et al. 2005; Stark et al. 2006) while others proposed that ice grains are fractal-like or fluffy aggregates (Praburam \& Goree 1995; Okuzumi 2009; Matthews et al. 2012). 
Table 1

Nominal Parameters for an Ice Dusty Plasma in Argon Background Gas

\begin{tabular}{lccc}
\hline \hline & Symbol & Nominal Value & Units \\
\hline Background gas density & $n_{\mathrm{g}}$ & $2 \times 10^{15}$ & $\mathrm{~cm}^{-3}$ \\
Water vapor density & $n_{\mathrm{H}_{2} \mathrm{O}}$ & $<2 \times 10^{13}$ & $\mathrm{~cm}^{-3}$ \\
Ion density & $n_{\mathrm{i}}$ & $10^{10}$ & $\mathrm{~cm}^{-3}$ \\
Ionization fraction & $n_{\mathrm{i}} / n_{\mathrm{g}}$ & $5 \times 10^{-6}$ & $\ldots$ \\
Dust grain density & $n_{\mathrm{d}}$ & $2 \times 10^{4}$ & $\mathrm{~cm}^{-3}$ \\
Background gas temperature & $T_{\mathrm{g}}$ & 180 & $\mathrm{~K}$ \\
Ion temperature & $T_{\mathrm{i}}$ & 180 & $\mathrm{~K}$ \\
Electron temperature & $T_{\mathrm{e}}$ & 20,000 & $\mathrm{~K}$ \\
Dust grain major radius & $b_{\mathrm{d}}$ & 30 & $\mu \mathrm{m}$ \\
Dust grain minor radius & $a_{\mathrm{d}}$ & 8 & $\mu \mathrm{m}$ \\
Dust grain charge number & $Z_{\mathrm{d}}$ & $6 \times 10^{4}-3 \times 10^{6}$ & $\cdots$ \\
RF input power & $P_{\mathrm{rf}}$ & 1 & $\mathrm{~W}$ \\
\hline
\end{tabular}

Notes. Much larger grains have been obtained by changing background gas pressure or species. Dust grain charge has been estimated by several methods; these methods are described in the Appendix. The smallest estimate for $Z_{\mathrm{d}}$ is obtained from the fact that electrostatic force exceeds gravitational force $\left(Z_{\mathrm{d}} e E>m_{\mathrm{d}} g\right)$. The largest estimate is obtained from the dispersion relation of dust-acoustic wave, $\omega / k=\left(Z_{\mathrm{d}} k_{\mathrm{B}} T_{\mathrm{i}} / m_{\mathrm{d}}\right)^{1 / 2}$. An ellipsoid capacitor model, $e Z_{\mathrm{d}}=C V_{\mathrm{d}}$ where $C$ is the capacitance of an ellipsoid and $V_{\mathrm{d}}$ is the dust floating potential, gives an estimate that is in between the gravity-based and wavebased estimates.

The shape of ice grains is important because the shape affects ice grain interaction with both electromagnetic radiation and background gas. Randomly polarized starlight can be (linearly) polarized after being scattered by elongated, aligned ice grains; this can explain the observed strong light polarization from protoplanetary disks (Gupta et al. 2005; Tanii et al. 2012). As another example of the importance of dust grain shape, Okuzumi (2009) argue that fractal shapes reduce the degree of ionization of protoplanetary disks.

On considering scales within the solar system, Saturn's outer rings such as E-ring, F-ring, and G-ring are water-ice dusty plasmas, as are the B-ring spokes. These dusty plasmas are composed of negatively charged, micron-size water-ice grains and ionized gas (Goertz 1989). Saturn's icy moon, Enceladus, has attained recent attention after the Cassini spacecraft measured an icy plume that populates Saturn's E-ring with water (Porco et al. 2006). At a still smaller scale, comet tails consist of ionized gas and water-ice (Davies et al. 1997) with the water-ice grains charged positively by photoemission of electrons by sunlight.

On the even smaller terrestrial scale, polar mesospheric clouds form during summertime at $85 \mathrm{~km}$ altitude (mesopause) in Earth's polar regions and are composed of weakly ionized plasma and water-ice grains. Sounding rocket measurements show that the water-ice grain size ranges between 10 and $100 \mathrm{~nm}$ and the grains are mostly negatively charged (Havnes et al. 1996). The observed depolarization of polarized lidar from mesospheric clouds (Baumgarten \& Fricke 2002) suggests that these grains are non-spherical.

\subsection{Caltech Icy Dusty Plasma Experiment}

We report here results from a laboratory experiment where water-ice grains are produced in a weakly ionized, lowtemperature plasma environment. Experimental parameters for a nominal argon plasma are listed in Table 1. Much larger and much smaller dust grains than in Table 1 can be obtained by adjusting experimental parameters.

An important result of this type of experiment (Shimizu et al. 2010; Chai \& Bellan 2014) is that water-ice grains form spontaneously when water molecules exist in a weakly ionized plasma having very cold background neutral gas, a situation of relevance to astrophysical situations. The formation of the water-ice grains in the lab plasma is also homogeneous, i.e., it does not require any non-ice nucleating material. The spontaneous, homogeneous formation requires existence of plasma; without plasma, water-ice grains do not form. These observations should be of substantial relevance to astrophysical situations, as they contradict current models of water-ice grain formation which typically assume that (1) nucleation is inhomogeneous, (2) ice grains are spherical, and (3) the density of ice grains follows a power law $n \sim a^{-p}$. Our previous experiments were carried out in a weakly ionized argon plasma. We report here how changing the background gas species affects water-ice grain growth and morphology. We also report results on the creation of non-water ices in weakly ionized plasma. While the lab experiment provides many insights, we do not claim that the lab plasma is an exact replica of any particular astrophysical situation. In fact, the number densities of neutrals, ions, and ice grains are all substantially larger than the corresponding densities in astrophysical dusty plasmas. We believe that these differences in densities will mainly lead to differences in timescales so growth that takes a few minutes in the lab would typically take orders of magnitude longer time in the much more diffuse astrophysical situation.

\section{EXPERIMENTAL SETUP}

Detailed information about the experimental design and operation is described in Chai \& Bellan (2013) and is briefly summarized here. Two electrically floating, parallel-plate aluminum electrodes in a small vacuum chamber have cold fingers extending out of the vacuum chamber.

The electrodes are cooled down for 30-40 minutes by immersing these cold fingers in liquid nitrogen where they remain during the experiment. After the initial cool down, a controlled amount of hydrogen, helium, neon, argon, or krypton gas is introduced into the vacuum chamber; this gas is cooled by contact with the cooled-down electrodes. A 13.56 MHz, $\sim 1 \mathrm{~W}$ rf generator is then connected across the electrodes to create a weakly ionized plasma in the hockeypuck sized region between the electrodes. Immediately after plasma ignition, a controlled amount of water vapor is introduced into the chamber using a leak valve. Water-ice grains then spontaneously form, grow, and levitate in the region between the two electrodes. Since nucleation only takes place in a narrow window of parameter space, we vary background gas pressure, rf power, and the amount of water vapor after nucleation occurs; we effectively separate the growth process from the nucleation process. What limits the nucleation of water-ice grain is now under investigation. The size, shape, and density of the water-ice grains depend on these external parameters.

Figure 1 shows the optical system used to image the waterice grains. A $500 \mathrm{~W}$ halogen lamp is directed to shine highintensity white light through a window on one side of the vacuum chamber. A long-distance microscope lens (QM-100; Questar) mounted on a digital SLR camera (D5300; Nikon) is 


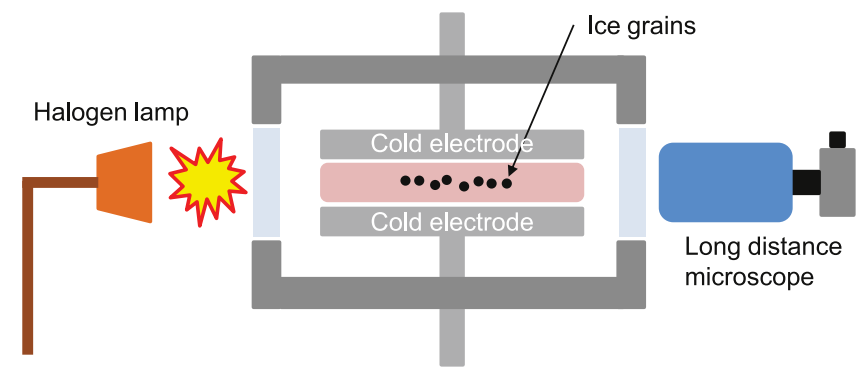

Figure 1. Sketch of long-distance microscope system. Halogen lamp provides backlight while digital SLR camera and microscope lens capture magnified images of water-ice grains.

placed to look through a window on the opposite side of the vacuum chamber. The backlit water-ice grains in the plasma are thus imaged on the camera. The nominal distance between the center of the vacuum chamber and the entrance aperture of the long distance microscope lens is $15-20 \mathrm{~cm}$, the magnification is approximately 10 times, and the depth of field is $30 \mu \mathrm{m}$. Since the pixel size of the Nikon D5300 is $3.9 \mu \mathrm{m}$, one camera pixel corresponds to $0.4 \mu \mathrm{m}$. To avoid image blurring and achieve high resolution, a $250 \mu$ s exposure time with low International Standards Organization number $(\leqslant 400)$ is used. The short exposure time is why a strong light source is needed. Spatial resolution of this system is about $3 \mu \mathrm{m}$. Because only ice grains within the $30 \mu \mathrm{m}$ depth of field form sharp images, the system effectively photographs a sheet region $30 \mu \mathrm{m}$ thick. Movie cameras have been used to track dust grain motion.

In addition to photography, Fourier transform infrared (FTIR) spectroscopy is used to determine whether the waterice grains are crystalline or amorphous. The ice grains are again illuminated by the intense halogen light but now the long distance microscope lens and camera are replaced by a FTIR spectrometer (Arcoptix FT-MIR Rocket 2.0-6.0). To allow transmission of infrared radiation, the vacuum chamber windows are sapphire and a $\mathrm{CaF}_{2}$ lens $(D=25 \mathrm{~mm}$, $f=50 \mathrm{~mm}$ ) focuses the radiation passing through the ice grains onto an optical fiber (core $D=500 \mu \mathrm{m})$ transparent in the $1-6 \mu \mathrm{m}$ infrared range. By measuring the transmitted infrared light passing through the ice grains as a function of wavelength, this system gives the ice infrared absorption spectrum.

\section{RESULTS}

Figures 2(a)-(e) show morphology of water-ice grains generated in hydrogen, helium, neon, argon, and krypton plasmas, respectively. Figures $2(\mathrm{a})-(\mathrm{d})$ are collages made from several different photos because the individual grains are too far apart from each other to make an image showing both the dust grain size and the inter-grain spacing. In contrast, Figure 2(e) is a single photograph of many adjacent grains and shows both the size of an individual grain and the intergrain spacing. The scale bar in Figure 2(a) applies to all of Figures 2(a)-(e). The input water vapor flow and the input rf power were fixed for these images but the ambient gas pressures differed such that the pressures of the background gas in the $\mathrm{H}, \mathrm{He}, \mathrm{Ne}, \mathrm{Ar}$, and $\mathrm{Kr}$ plasmas were respectively 240 , 300, 300, 100, and 100 mTorr. In Chai \& Bellan (2013) we showed that larger ice grains are formed at lower background gas pressure. The background gas pressures used in Figures 2(a)-(e) are the lowest pressures that could be used for each gas and so correspond to the largest ice grains that could be created for each gas. Figures $2(\mathrm{a})-(\mathrm{e})$ clearly show that larger and more complex ice grains form in plasmas having lighter background gas, i.e., the ice grains in hydrogen and helium plasmas are larger and more complicated in structure than the ice grains in krypton. It is further found that ice grains formed in hydrogen and helium plasmas have a self-similar, fractal nature. These grains are principally composed of Yshaped branches which, in turn, are composed of smaller-scale Y-shaped branches.

Figures 3(a) and (b) show how elongated ice grains levitate in the He and Ne plasmas and line up in the vertical direction. We observed such vertical alignment of elongated dust grains in most of our plasmas. Another interesting feature is that all the ice grains are regularly spaced from each other. This indicates a strong repulsive force between adjacent ice grains.

The average ice grain length as determined from the images is given in Figure 4(a); note that the $x$-axis is log scale. Figure 4(a) indicates that ice grain length increases as the particle mass of the background mass decreases. For example, the average ice grain length in a hydrogen plasma is $307 \mu \mathrm{m}$, 11 times longer than the $27 \mu \mathrm{m}$ average length of ice grains in krypton plasma. Aspect ratio, defined as the maximum length divided by the maximum width, is plotted in Figure 4(b). This shows that aspect ratio saturates at just under 5 as background gas atomic mass decreases. Circularity, defined as $4 \pi A / P^{2}$ where $A$ is the surface area of ice grain and $P$ is the perimeter of ice grain, is plotted in Figure 4(c). Circularity is a measure of ice grain roughness: 0 means very rough while 1 means smooth like a circle. Figure 4(c) shows that circularity decreases as background gas mass decreases, i.e., the ice grains become fluffier when generated in a plasma having lighter mass background gas.

Figures 5(a)-(e) show respectively the size (length) distributions of ice grains formed in $\mathrm{H}, \mathrm{He}, \mathrm{Ne}, \mathrm{Ar}$, and $\mathrm{Kr}$ plasmas. It is clearly seen that all the distributions do not have a power law dependence on grain length; they are rather similar to mono-disperse or log-normal although the number of samples is too small to obtain a statistical conclusion.

The fractal dimension of ice grains is calculated as follows: (1) the grain perimeter $N$ is measured using a ruler of length $R$; (2) the perimeter is then repeatedly measured using smallerscale rulers such as $R / 2, R / 4, R / 8, \ldots R / S$, where $S$ is the scale factor; (3) $\log N$ is plotted versus $\log S$ and a linear function is fitted to obtain the slope, i.e., fractal dimension $\left(N=S^{D}\right)$. Figure 6 shows plots of $\log N$ versus $\log S$ and linear fits for hydrogen, helium, neon, argon, and krypton plasmas, respectively. The obtained fractal dimensions are $1.75,1.71,1.63$, 1.68 , and 1.62, indicating higher ice grain fractal dimension in the lighter background gas plasmas. This implies that the boundary of ice grains formed in the lighter gas plasmas tends to be more two-dimensional than one-dimensional.

Figure 7 shows mid-infrared absorption spectra obtained from the FTIR spectrometer. Solid, dashed, dotted, and dashed-dotted lines represent respectively $\mathrm{He}, \mathrm{Ne}, \mathrm{Ar}$, and $\mathrm{Kr}$ plasmas. The spectra have peaks at $\sim 3140, \sim 3240$, and $\sim 3360 \mathrm{~cm}^{-1}$. Except for $\mathrm{He}$, the absorption spectra have a distinct $3240 \mathrm{~cm}^{-1}$ peak (O-H stretch), indicating the ice grains are crystalline (Mastrapa et al. 2009) as has been observed in protoplanetary disks (McClure et al. 2012), Saturn's rings (Poulet et al. 2003; Porco et al. 2006), and polar mesospheric clouds (Turco et al. 1982). For ice grains in He plasma at early 


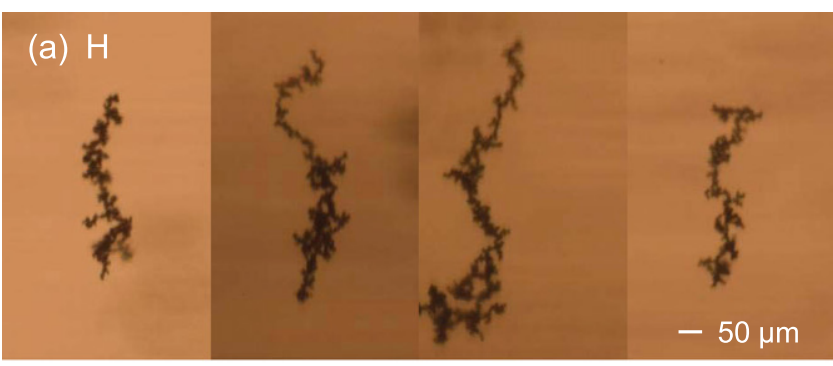

(b) $\mathrm{He}$
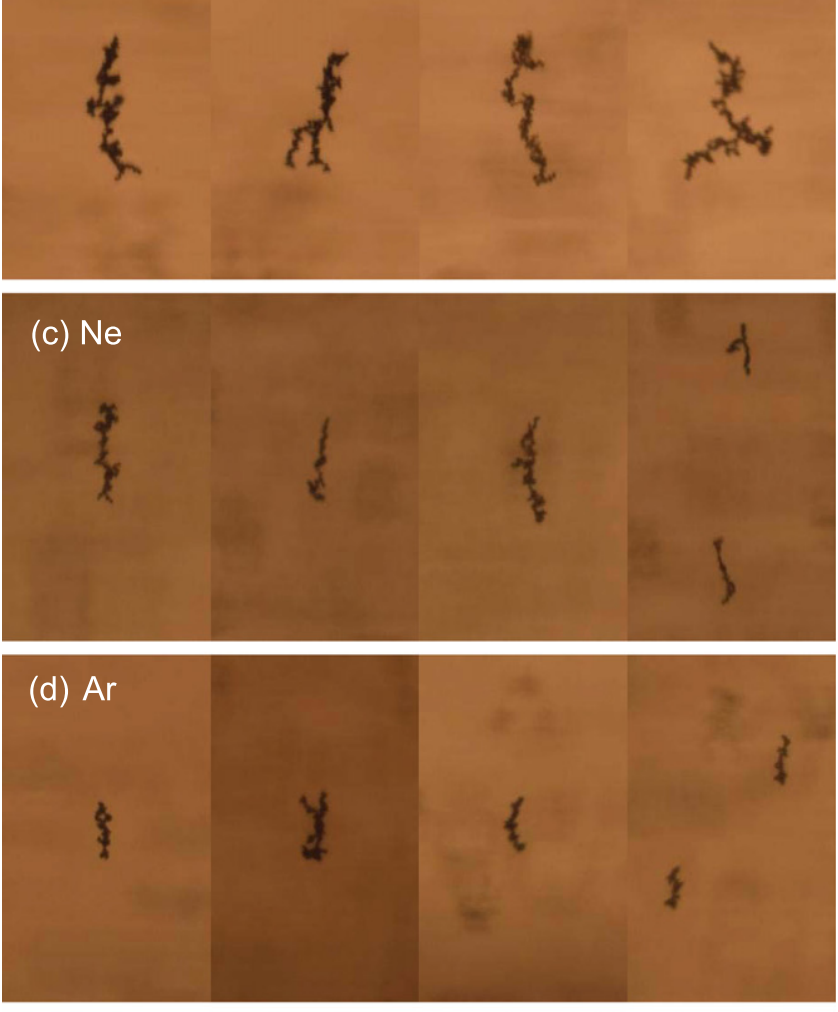

(e) $\mathrm{Kr}$

Figure 2. Water-ice grains formed in various gas plasmas. (a)-(e) are respectively obtained from $\mathrm{H}, \mathrm{He}, \mathrm{Ne}, \mathrm{Ar}$, and $\mathrm{Kr}$ plasmas. Figures $2(\mathrm{a})-(\mathrm{d})$ are composed of several images due to low number density of ice grain. Larger and more complex-structured ice grains are formed in lighter gas plasmas.

times, i.e., when ice grains are small, the absorption spectrum has a distinct peak at around $3240 \mathrm{~cm}^{-1}$ just like the $\mathrm{Ne}$ spectrum. However, as the He plasma ice grains grow, this distinct $3240 \mathrm{~cm}^{-1}$ peak disappears and the spectrum broadens toward low wave number direction; this broadening is attributed to scattering from ice grains (Clapp et al. 1995).

The position of the $3240 \mathrm{~cm}^{-\mathrm{P}}$ peak can be used to deduce the temperature at which the ice grains form (Clapp et al. 1995). The measured peak position ranges between
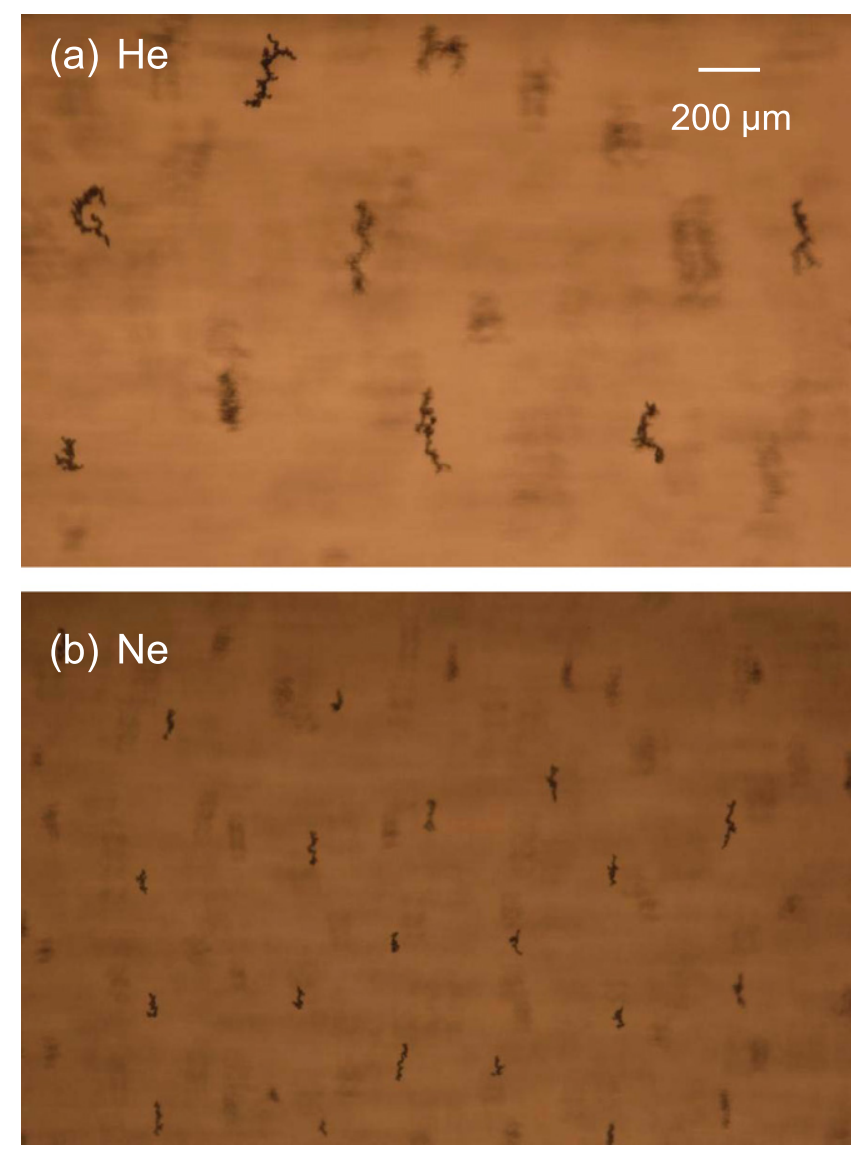

Figure 3. Alignment of elongated water-ice grain in (a) $\mathrm{He}$ and (b) $\mathrm{Ne}$ plasmas. Water-ice grains are regularly spaced.

3236 and $3240 \mathrm{~cm}^{-1}$ indicating a temperature $\sim 180 \mathrm{~K}$. This temperature is similar to the temperatures of several astrophysical dusty plasmas.

It was also determined that the water vapor pressure is much lower than the 1-2 mTorr previously reported in Chai \& Bellan (2014). The water vapor pressure was previously measured by a capacitance manometer mounted on a vacuum port quite far from the plasma. The temperature at the capacitance manometer was near room temperature and it was realized that the water vapor pressure at the manometer would be substantially higher than that in the much colder plasma region. We tried to measure the water vapor density by measuring the absorption of a diode laser (EP1392-DM-B01; Eblana) tuned to the $1392 \mu \mathrm{m}$ water vapor molecular absorption line. This system can detect $\mathrm{H}_{2} \mathrm{O}$ partial pressure as low as partial pressure as $5 \mathrm{mTorr}$ at room temperature. The laser absorption line disappeared immediately after the cooling process started, indicating a probable water vapor pressure much less than $1 \mathrm{mTorr}$ at the operation temperature.

Besides water-ice grains, we also successfully created ice grains with acetone and methanol as shown in Figure 8. It is found that both acetone and methanol ice grains are not spherical but are elongated as observed for water-ice grains. On the other hand, we could not obtain ice grains with carbon dioxide.

Figure 9 shows an FTIR spectrum obtained from methanol ice grains formed in a 500 mTorr argon plasma. Comparison of the shape of this spectrum to Figure 3 of Lucas et al. (2005) 

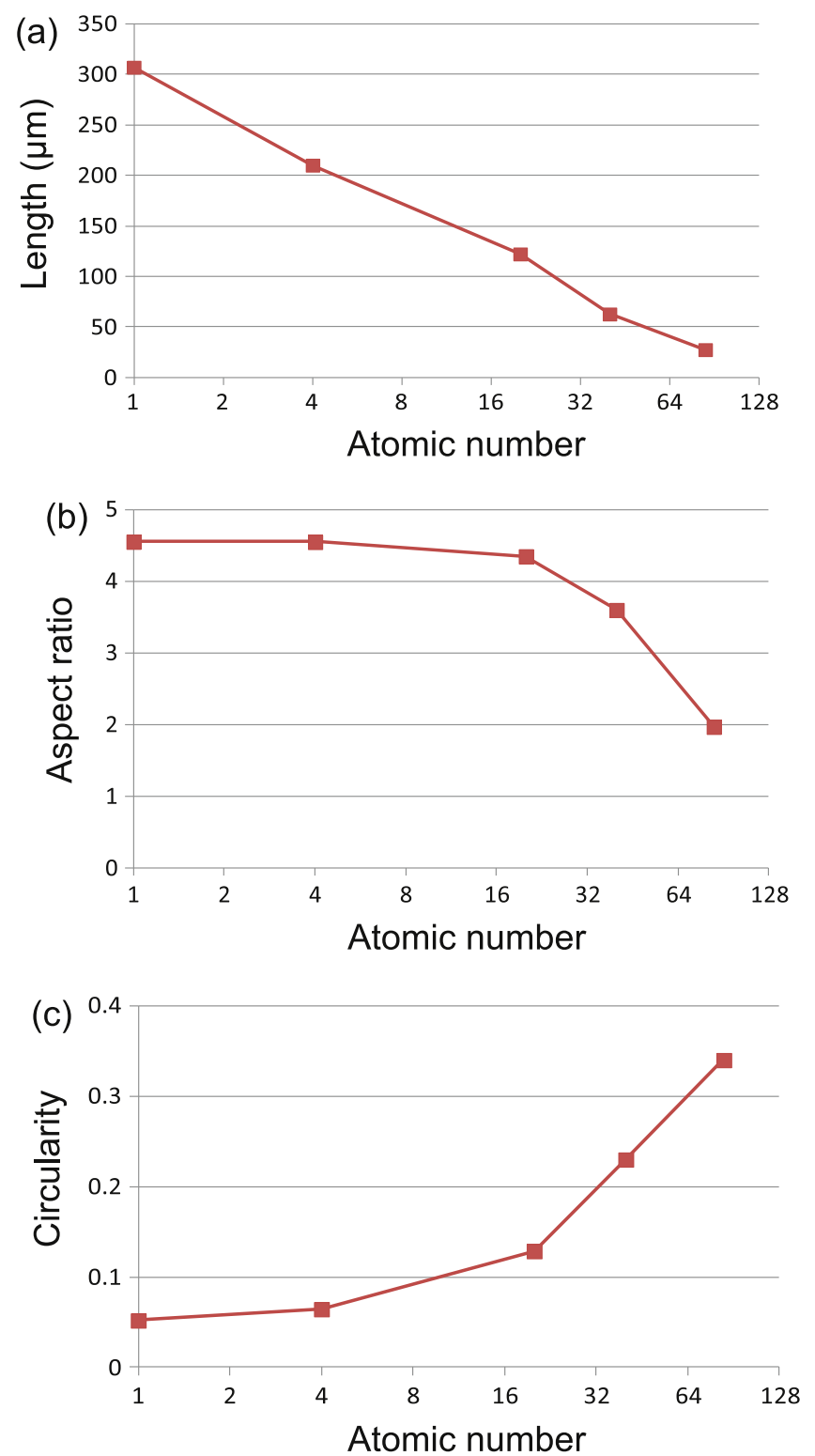

Figure 4. (a) Length, (b) aspect ratio, and (c) circularity of ice grains as a function of background gas mass. As background gas mass decreases, length and aspect ratio increase (aspect ratio saturates at $\sim 5$ ) while circularity decreases (becomes fluffier).

suggests that the methanol ice grains are in the beta-crystalline phase which forms at $\sim 170 \mathrm{~K}$.

\section{DISCUSSION}

Treatments of astrophysical dust typically assume that dust grows by agglomeration of cluster particles (Okuzumi 2009; $\mathrm{Du}$ et al. 2010; Matthews et al. 2012). Since the cluster particles are usually charged with the same polarity, an interparticle repulsive force would then have to be overcome if there is to be any agglomeration and so some mechanism must be invoked to provide sufficient kinetic energy to overwhelm the mutual repulsion. Some authors argue turbulence provides such energy (Okuzumi 2009; Matthews et al. 2012) while others argue that dust-acoustic waves would do this (Du et al. 2010). Using a high speed movie camera we investigated whether the ice dust grains in our plasmas collide with each other but have been unable to observe any evidence of collisions; instead the ice grains always remain well-separated from each other as shown in the animations associated with Figures 10 and 11. However, since our camera system is only able to detect particles larger than $2 \mu \mathrm{m}$, it is possible that sub-micron particles could collide, but this would require a size-dependant collision mechanism favoring small particles.

From this observation we believe that the ice grain growth mechanism in our experiment is unlikely to involve ice grains colliding with each other. Instead, the behavior of the dust grains in our experiment is consistent with the following growth mechanism. Water molecules located within the screening length of a negatively charged ice dust grain are preferentially attracted to the sharp edges of the ice grain, i.e., to the two ends of an ellipsoidal dust grain. This is because the large dipole moment of water molecules causes them to be attracted to regions of strong electric field. In order for a water molecule to reach the ice grain surface and be captured, the water molecule should not be deflected by a collision with a background gas molecule. It should be recalled that the density of background gas molecules is at least two orders of magnitude higher than the density of water molecules and so water molecules mainly collide with background gas molecules. Thus, collision with a background gas molecule makes it more difficult for a water molecule to reach the ice grain than if there is no collision. This argument is consistent with Chai \& Bellan (2014) where it was observed that ice grains grow larger and more elongated at low ambient pressure, i.e., when there are fewer collisions with background molecules. The argument differs in substance from commonly invoked ideal gas models (e.g., Hesstvedt 1961, Hertz-Knudsen equation) which predict that the growth rate of ice grains depends only on the water vapor partial pressure and not on the pressure of the ambient, non-water gas molecules. The observed disagreement with ideal gas models provides strong support for the presumption that ice-grain growth involves the collisionless, orbital behavior of water molecules in the electric field produced by charged ice grains.

The strong dependence on the mass of the background gas molecules provides further support for the deflection model described above because of the following argument. Suppose a water molecule collides with a background gas molecule while being attracted to the large electric field gradient at the end of an ellipsoidal ice grain. Since the ambient gas molecules move at their nominal thermal velocity, whereas the water molecule is accelerated by the electric field gradient to a higher than thermal velocity, it is reasonable to approximate the background gas molecule as being stationary. If the background gas molecule is much lighter than the water molecule, the water molecule scatters little after having a collision with the background gas molecule and so can stay on its course toward its targeted position on the ice grain. On the other hand, if the background gas molecule is much heavier than the water molecule, the water molecule is scattered by a large angle and is deflected from its initial course. Thus larger ice grains form in light background gas plasmas (e.g., $\mathrm{H}, \mathrm{He}$ ) than in heavy background gas plasmas (e.g., Ar, Kr).

Another result supporting the deflection model is that we have produced ice grains using acetone or methanol but have been unsuccessful when trying carbon dioxide. This may 

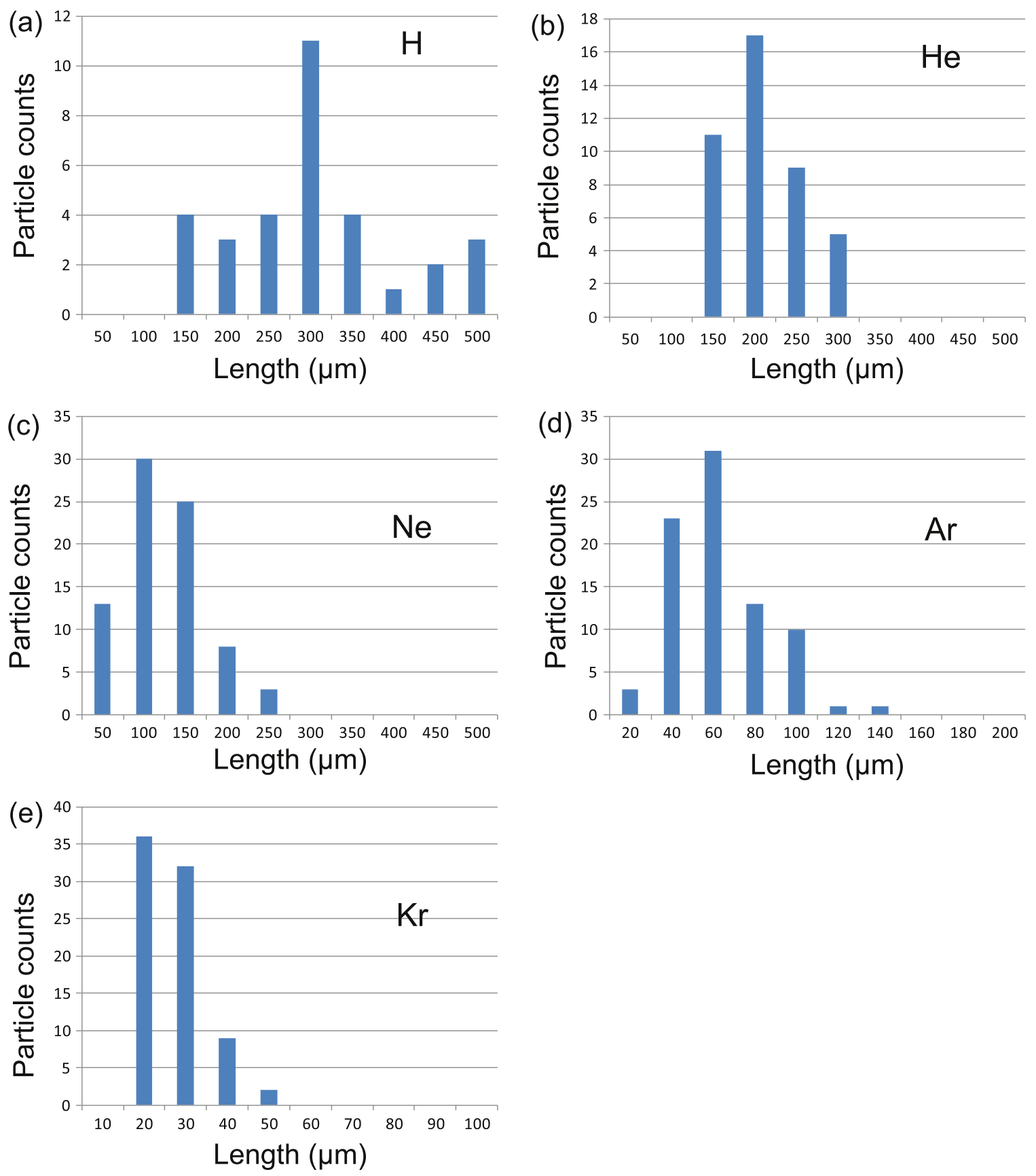

Figure 5. Size distributions of ice grains created in (a) H, (b) He, (c) Ne, (d) Ar, and (e) Kr plasmas, indicating that ice grains are closer to mono-dispersive than power law distribution.

endorse the premise that high polarity of the incident molecule is critical because acetone (2.91 Debye) and methanol (1.7 Debye) have dipole moments larger than or similar to water molecules (1.85 Debye) whereas carbon dioxide has zero dipole moment. Alternatively, it could be related to the fact that carbon dioxide has much higher saturated vapor pressure $(\sim 1$ Torr) than water, acetone, or methanol ( $<1 \mathrm{mTorr})$ at $180 \mathrm{~K}$ and so we are only able to provide unsaturated carbon dioxide gas which makes nucleation of carbon dioxide ice extremely difficult.

Water-ice grains immersed in a laboratory plasma experience several forces such as electrostatic force, ion drag force, thermophoretic force, neutral drag force, and gravitational force. The electrostatic force arises from the negatively charged ice grains being in the presence of the sheath electric field near the electrodes. Ion drag force is the force resulting from ions being accelerated by the sheath electric field interacting with the ice grains. Since the ions are positively charged, their flow direction and hence the force they exert is in the opposite direction of the direct electric force on the ice grains. The thermophoretic force results from differential momentum transfer associated with a temperature gradient in the background gas. Neutral drag force is the force that arises from gas flow of the background gas. Ice grains levitate at a position where all these forces balance, i.e., at a minimum of the total potential energy resulting from all these forces. 

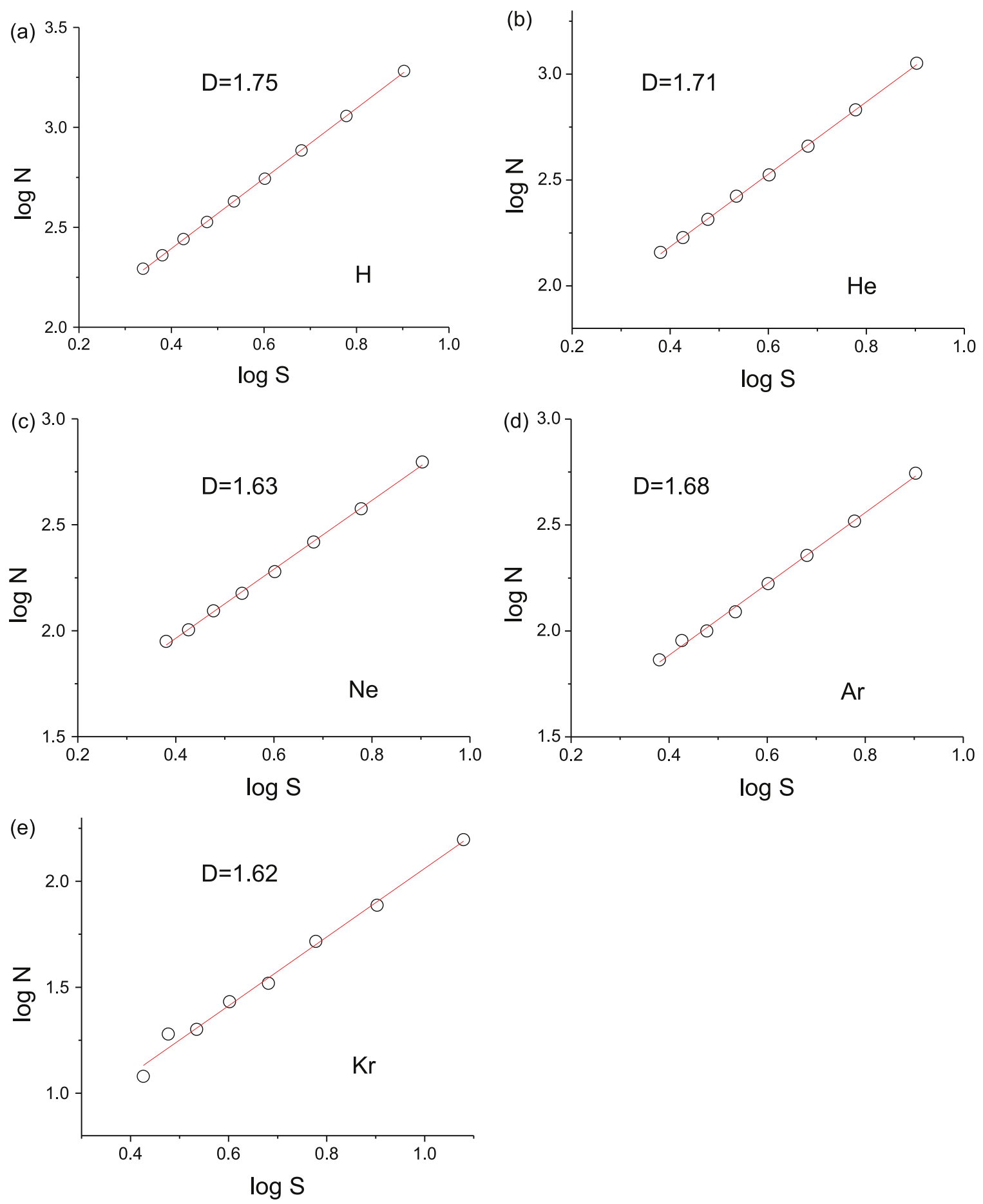

Figure 6. $\log N-\log S$ plots for obtaining fractal dimension of ice grains formed in (a) $\mathrm{H}$, (b) $\mathrm{He}$, (c) $\mathrm{Ne}$, (d) $\mathrm{Ar}$ and (e) $\mathrm{Kr}$ plasmas. Fractal dimensions are 1.75, 1.71, $1.63,1.68$, and 1.62 for ice grains formed in $\mathrm{H}, \mathrm{He}, \mathrm{Ne}, \mathrm{Ar}$, and $\mathrm{Kr}$ plasmas, respectively.

The electrostatic and ion drag forces are exerted along the electric field: the electrostatic force is exerted toward the interior of the bulk plasma while the ion drag force is exerted toward electrodes. This is because the plasma potential is positive as a result of electron confinement being worse than ion confinement in an unmagnetized plasma resulting in the creation of an ambipolar electric field oriented to drive negatively charged particles away from the electrodes. The thermophoretic force is presumed to be exerted toward electrodes because the bulk plasma will be warmer than the liquid nitrogen cooled electrodes. Gravitational force and neutral drag force are found to be negligible: the entire chamber was tilted to see if changing the direction of the gravitational force from being normal to the electrodes changes ice grain growth or levitation; no dependence on tilt angle was observed, indicating no dependence on gravitational force. The input gas flow rate was also varied to no effect. Because all the dominant forces are exerted along the vertical direction, ice 
Wavelength $(\mu \mathrm{m})$

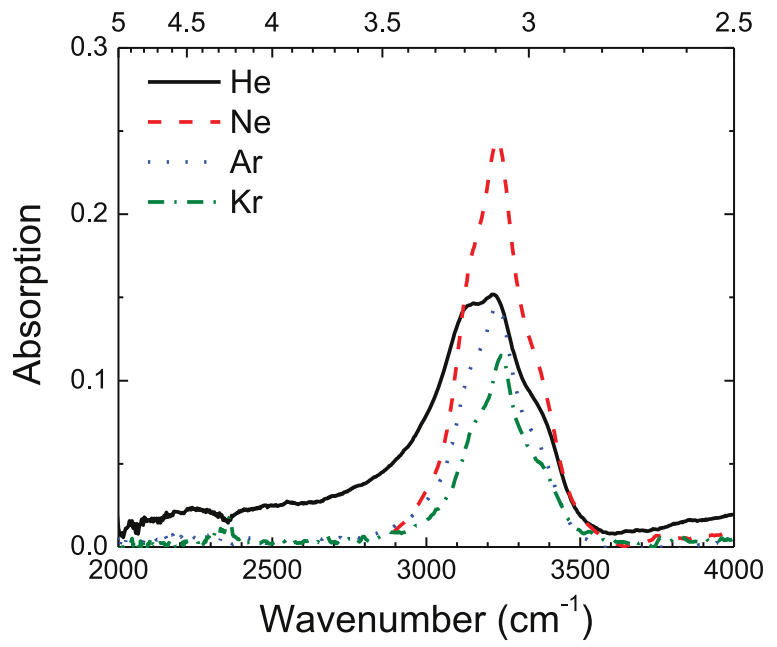

Figure 7. Absorption signals of water-ice grains measured by in situ FTIR. Solid, dashed, dotted, and dashed-dotted lines respectively represent $\mathrm{He}, \mathrm{Ne}$, $\mathrm{Ar}$, and $\mathrm{Kr}$ plasmas. Distinct peaks around $3240 \mathrm{~cm}^{-1}$ indicate crystalline composition.

(a)

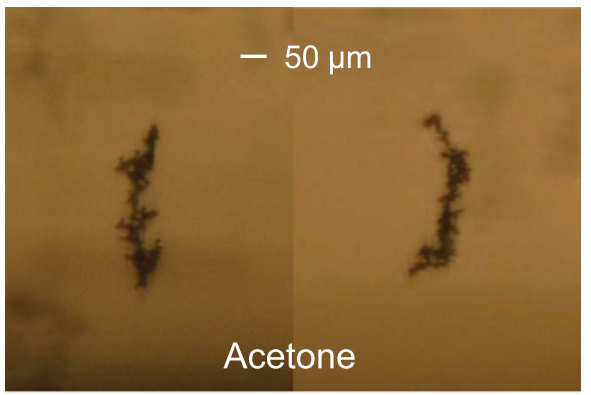

(b)

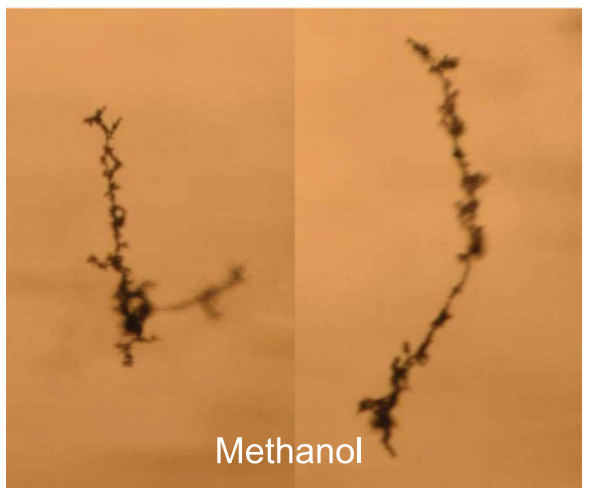

Figure 8. Composite images of (a) acetone ice grains and (b) methanol ice grains. Acetone ice grains forms in He 300 mTorr plasma while methanol ice grains forms in Ar 10 mTorr plasma.

grains having similar size and shape end up levitating in the same horizontal plane.

If the ice grain density is sufficiently large that the inter-grain distance is less than the Debye screening length, repulsive electrostatic forces occur between adjacent ice grains levitating in the same horizontal plane. This situation clearly occurs in our plasma: the Debye screening length is a few hundred $\mu \mathrm{m}$ while the interparticle distance is between a few hundred $\mu \mathrm{m}$ and a few $\mathrm{mm}$. The uniform spacing between ice grains in

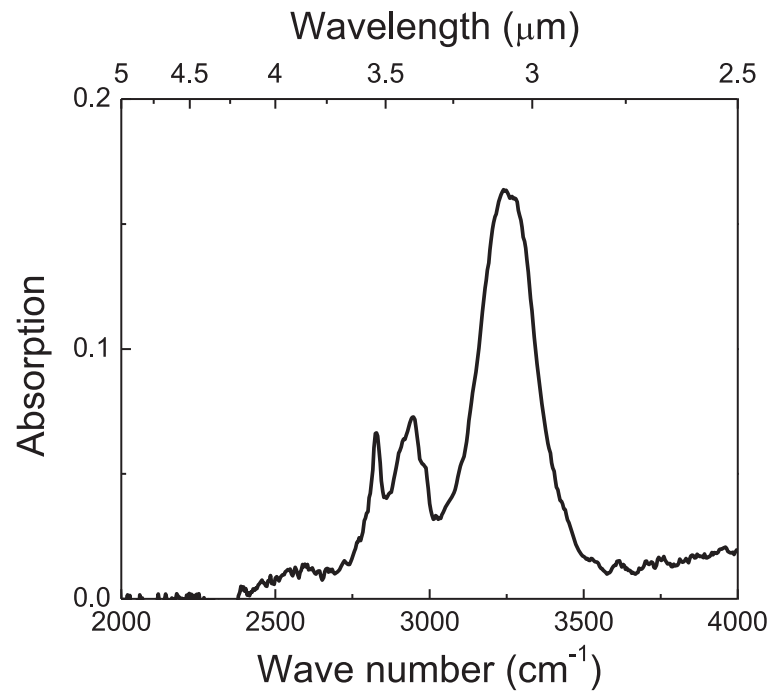

Figure 9. FTIR absorption spectrum of methanol ice grains in argon plasma with 500 mTorr neutral pressure.

Figure 3 clearly shows that a mutual repulsive force exists between ice grains.

Because the grains are elongated, as sketched in Figure 12 each has a charge $q / 2$ at one end and a charge $q / 2$ at the other end where $q$ is the total dust grain charge. Suppose as in Figure 12 that two identical grains levitate and are rotated at respective angles $\theta_{1}$ and $\theta_{2}$ relative to the $x$-axis. The electric potential energy $U$ associated with the mutual interaction of the four half-charges at the two ends of the two grains is

$$
\begin{aligned}
U= & \frac{(q / 2)^{2}}{4 \pi \varepsilon_{0}}\left[\left\{\left(\frac{L}{2}\left(\cos \theta_{1}+\cos \theta_{2}\right)-D\right)^{2}\right.\right. \\
& \left.+\left(\frac{L}{2}\left(\sin \theta_{1}+\sin \theta_{2}\right)\right)^{2}\right\}^{-1 / 2} \\
& +\left\{\left(\frac{L}{2}\left(\cos \theta_{1}-\cos \theta_{2}\right)-D\right)^{2}\right. \\
& \left.+\left(\frac{L}{2}\left(\sin \theta_{1}-\sin \theta_{2}\right)\right)^{2}\right\}^{-1 / 2} \\
& +\left\{\left(\frac{L}{2}\left(\cos \theta_{1}+\cos \theta_{2}\right)+D\right]^{2}\right. \\
& \left.+\left(\frac{L}{2}\left(\sin \theta_{1}+\sin \theta_{2}\right)\right)^{2}\right\}^{-1 / 2} \\
& +\left\{\left(\frac{L}{2}\left(\cos \theta_{1}-\cos \theta_{2}\right)+D\right]^{2}\right. \\
& \left.\left.+\left(\frac{L}{2}\left(\sin \theta_{1}-\sin \theta_{2}\right)\right)^{2}\right\}^{-1 / 2}\right]
\end{aligned}
$$

where $L$ is the ice grain length and $D$ is the distance between the grain midpoints and it is assumed that the grains are closer 


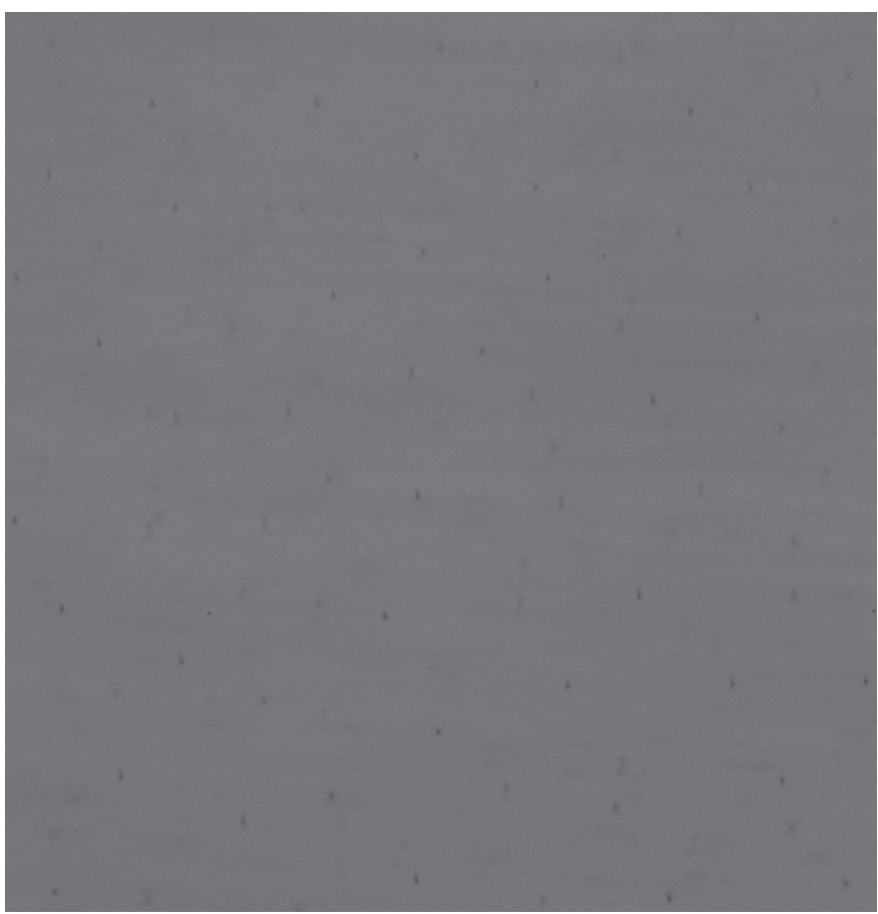

Figure 10. Movie of ice grain motion captured with $20 \mu$ s exposure time and $1000 \mathrm{fps}$ frame rate in the Ar 100 mTorr plasma by Photron Fastcam $\mathrm{U} \times 50$. Field of view recorded by this camers covers an area of $1.3 \mathrm{~mm}$ by $1.0 \mathrm{~mm}$. The displaying frame rate is $30 \mathrm{fps}$. It is clearly seen that the ice grains are regularly spaced and do not collidce with each other.

(An animation of this figure is available.)

than the shielding distance. By defining $x=L / D$, this potential energy can be expressed using $\cos \theta_{1} \cos \theta_{2}+\sin \theta_{1} \sin \theta_{2}=$ $\cos \left(\theta_{1}-\theta_{2}\right)$ as

$$
\begin{aligned}
U= & \frac{(q / 2)^{2}}{4 \pi \varepsilon_{0} D}\left[\left\{1-\left(\cos \theta_{1}+\cos \theta_{2}\right) x\right.\right. \\
& \left.+\frac{1}{2}\left[1+\cos \left(\theta_{1}-\theta_{2}\right)\right] x^{2}\right\}^{-1 / 2} \\
& +\left\{1-\left(\cos \theta_{1}-\cos \theta_{2}\right) x\right. \\
& \left.+\frac{1}{2}\left[1-\cos \left(\theta_{1}-\theta_{2}\right)\right] x^{2}\right\}^{-1 / 2} \\
& +\left\{1+\left(\cos \theta_{1}+\cos \theta_{2}\right) x\right. \\
& \left.+\frac{1}{2}\left(1+\cos \left(\theta_{1}-\theta_{2}\right)\right) x^{2}\right\}^{-1 / 2} \\
& +\left\{1+\left(\cos \theta_{1}-\cos \theta_{2}\right) x\right. \\
& \left.\left.+\frac{1}{2}\left(1-\cos \left(\theta_{1}-\theta_{2}\right)\right) x^{2}\right\}^{-1 / 2}\right]
\end{aligned}
$$

Because $x \ll 1$ in our experiment, the above equation simplifies to

$$
U=\frac{q^{2}}{8 \pi \varepsilon_{0} D}\left(8+\left(3 \cos ^{2} \theta_{1}+3 \cos ^{2} \theta_{2}-2\right) x^{2}\right)
$$

using $\left(1+a x+b x^{2}\right)^{-1 / 2} \approx 1-a x / 2+\left(3 a^{2} / 8-b / 2\right) x^{2}$. Thus, the two ice grains have minimum potential energy when

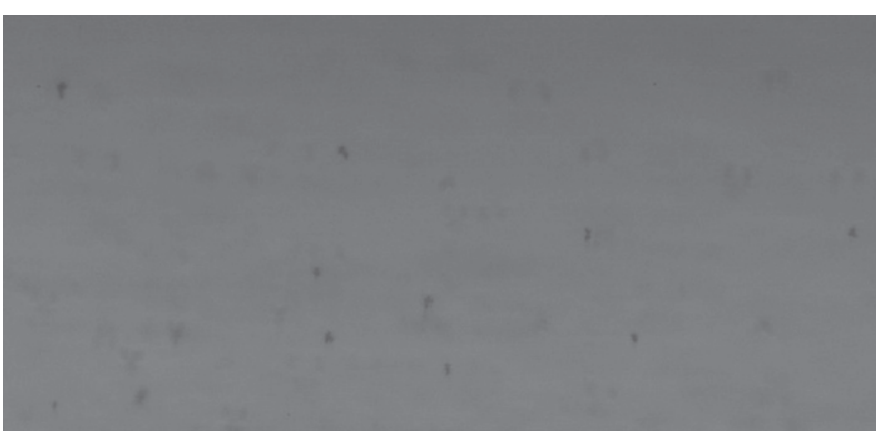

Figure 11. Movie of ice grain motion where dust-acoustic wave occurs. This was captured with $20 \mu$ s exposure time and $2000 \mathrm{fps}$ frame rate in the $\mathrm{Ar}$ 100 mTorr plasma. Field of view covers are area of $1.3 \mathrm{~mm}$ by $0.5 \mathrm{~mm}$. The displaying frame rate is $5 \mathrm{fps}$. No collisions between ice grains are observed.

(An animation of this figure is available.)

$\theta_{1}=\theta_{2}=90^{\circ}$, indicating vertical alignment is favorable. This explains why ice grains are all lined up in the vertical direction, i.e., along the electric field direction.

We postulate that elongated, fractal-like ice grains exist in a variety of astrophysical dusty plasma situations because:

1. both our plasma and astrophysical dusty plasmas are in the collisionless regime for water molecules near the dust grain, i.e., the mean free path of water molecule is larger than the ice grain screening length,

2. both our plasma and astrophysical dusty plasmas are weakly ionized so the arguments regarding collisions with background gas will be similar,

3. astrophysical dusty plasmas typically consist of relatively light ambient gases; for example, protoplanetary disks are mainly composed of light gas such as $\mathrm{H}$ and $\mathrm{He}$ and Saturn's rings consist of $\mathrm{O}$ and $\mathrm{OH}$ (Ip 2000) while polar mesospheric clouds are composed of $\mathrm{N}_{2}$ gas,

4. the $\sim 180 \mathrm{~K}$ gas temperature in our experiment is similar to astrophysical situations.

While densities of background gas, water molecules, plasma, and dust grains are much higher in our experiment than in typical astrophysical situations, the ratios of these various densities are similar. We believe the difference in absolute densities will result in much slower growth rates and possibly different dust grain sizes but otherwise will not change the phenomenology. Our previous measurement of growth rate of water-ice grains supports this argument (Chai \& Bellan 2014): the growth rate is proportional to water vapor partial pressure and input rf power (i.e., plasma density) and inversely proportional to ambient gas pressure. The fact that growth rate is proportional to water vapor partial pressure and inversely proportional to ambient gas pressure is reasonable because water vapor is the source of material for the ice grains and because collisions between water molecules and ambient gas impede ice grain growth. However, we do not know how plasma density affects the ice grain growth rate although it presumably determines the screening length (Debye length) of ice grains. Alignment of elongated grains may occur in some astrophysical plasmas where the ice grain density is relatively high so the Coulomb interaction between ice grains becomes important.

A significant effect of dust grain fractal and non-spherical morphology is a large surface to volume ratio so the grains can adsorb more surrounding gas atoms than the same mass of 


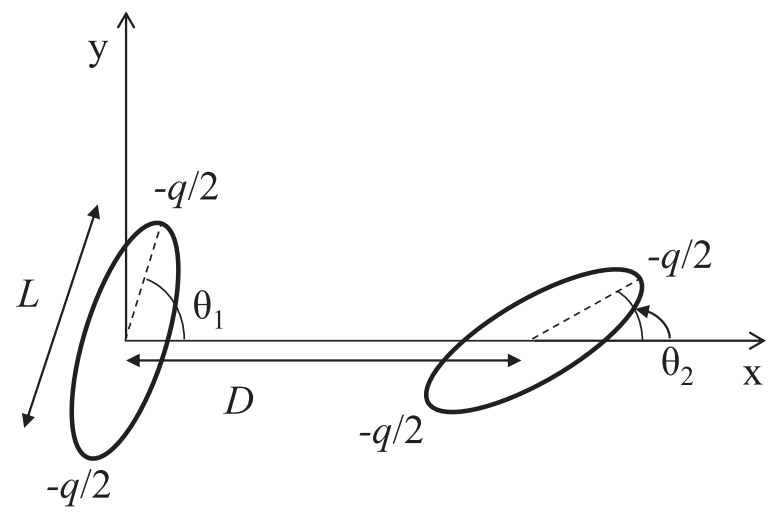

Figure 12. Two randomly oriented, identical, elongated ice grains. Because they are elongated, half the charge resides on one end and half on the other end.

spherical ice. Also, the dynamics would differ from a sphere. For example, forces depending on ice grain surface area such as thermophoretic force, ion drag force, and gas drag force become relatively more important.

Amorphous water-ice exists in the outer regions of protoplanetary disks and in the interstellar medium because these situations are colder and amorphous ice grains forms at low temperature while crystalline ice grains forms at high temperature. The critical temperature depends on the pressure and other parameters. At present, the water-ice grains generated in our experiment are crystalline because the temperature is higher than the critical temperature for amorphous formation. We plan to operate at lower ambient gas temperatures in the future to see if amorphous ice grains can be produced; this will be monitored via the infrared absorption spectrum.

\section{CONCLUSION}

Our experiment and also that of Shimizu et al. (2010) demonstrate that ice grains form in the presence of weakly ionized plasma without the need for a non-ice nucleation center. This implies that ice may not be constrained to exist only in the form of a mantle on a refractory substrate.

The size and shape of the ice grains in our experiment is dictated by ambient conditions. This form of dependence implies that all the ice grains will have approximately the same size and shape as shown in Figure 5. This conclusion is very different from the presumption that ice grains are spherical and that ice grain number density has a power law dependence $\sim a^{-p}$ where $p=3.5$ results from an equilibrium balance of colliding ice grains fragmenting to become smaller and agglomerating to become bigger. Each ice grain size and shape in our experiment results from a balance between the ambient parameters that tend to make the grain larger (e.g., capture of water molecules) and the ambient parameters that tend to make the grain smaller (e.g., sublimation of water). Changing one ambient parameter (e.g., changing the mass or pressure of the background gas) changes all the ice grains the same way (e.g., lowering the background pressure makes all grains larger). The most significant parameters are the mass and pressure of background gas. Ice grains formed in low mass background gas plasmas such as hydrogen and helium are more elongated, larger, and more fractal-like than grains formed in high mass background gas. This is consistent with the scattering angle of incident water molecules from background gas molecules being proportional to the mass of the background gas molecule. Besides the size and shape, ice grains generated in our plasmas are crystalline and are elongated and aligned along the vertical direction, i.e., electric field direction. This mutual alignment is the lowest energy state for the Coulomb repulsive interaction between ice grains.

Our results suggest that water-ice grains in some astrophysical situations may not be spherical with a power law density dependence but instead are elongated, quasi-monodisperse, and fractal-like. Alignment of elongated ice grains may occur in astrophysical situations where the ice grain number density is sufficiently high for ice grains to produce mutual electrostatic forces.

This material is based upon work supported by the US Department of Energy Office of Science under Award Number DE-SC0010471. We thank J. Goree for graciously loaning the long distance microscope lens used in this work. We also thank S. Shimizu for kindly providing information on the design of his experimental apparatus. The Eblana laser was purchased using the Caltech Engineering and Applied Science Discovery Fund.

\section{APPENDIX \\ ESTIMATION OF ICE GRAIN CHARGE}

Although we do not have a direct way of measuring the ice grain charge $Z_{d}$, it can be estimated using the various ways described below.

Gravity method. Here, we treat an ice grain as a prolate ellipsoid having $a=b=8 \mu \mathrm{m}$ and $c=30 \mu \mathrm{m}$. Thus, the ice grain mass $m_{\mathrm{d}}=4 \pi \rho a b c / 3=7.4 \times 10^{-12} \mathrm{~kg}$. We have found that the electrostatic force exerted on an ice grain is stronger than the gravitational force: $e Z_{\mathrm{d}} E>m_{\mathrm{d}} g$. We can roughly estimate the sheath electric field by dividing the sheath potential by the sheath thickness: the sheath potential is $V_{s}=0.4 V_{\text {rf }}$ (Lieberman \& Lichtenberg 2005), where $V_{\text {rf }}$ is about $40 \mathrm{~V}$ and the sheath thickness is $s=2 \mathrm{~mm}$ obtained by measuring the thickness of the dark region near the electrode. The average sheath electric field is thus $E=V_{s}$ $/ s=8000 \mathrm{~V} \mathrm{~m}^{-1}$. This leads to $Z_{\mathrm{d}}>5.7 \times 10^{4}$.

Wave method. The dispersion relation of dust-acoustic wave is expressed as (Merlino et al. 1998)

$$
\frac{\omega}{k}=\left[\frac{k T_{\mathrm{d}}}{m_{\mathrm{d}}}+\left(1-\frac{n_{\mathrm{e}}}{n_{\mathrm{i}}}\right) Z_{\mathrm{d}} \frac{k T_{\mathrm{i}}}{m_{\mathrm{d}}} \frac{1}{1+\left(T_{\mathrm{i}} / T_{\mathrm{e}}\right)\left(n_{\mathrm{e}} / n_{\mathrm{i}}\right)}\right]
$$

Let us assume that a significant amount of electrons reside on the ice grains: $n_{\mathrm{e}} / n_{\mathrm{i}} \ll 1$. Then, the above equation can be rewritten as $\omega / k=\left(Z_{\mathrm{d}} k T_{\mathrm{i}} / m_{\mathrm{d}}\right)^{1 / 2}$. The phase velocity of a dust acoustic wave in a 100 mTorr of Ar plasma is measured to be $3 \mathrm{~cm} \mathrm{~s}^{-1}$ by a high speed movie camera, implying $Z_{\mathrm{d}}=2.7 \times 10^{6}$.

Capacitance method. Since the ice grain is much larger than an ion Debye length, the floating potential of the ice grain is given by

$$
V_{\mathrm{d}}=-T_{\mathrm{e}} \ln \left(\frac{m_{\mathrm{i}}}{2 \pi m_{\mathrm{e}}}\right)^{1 / 2}
$$

Using the Ar plasma parameters in Table 1 we obtain $V_{\mathrm{d}}=-9.4 \mathrm{~V}$. The dust grain is assumed to be a conductor so the charge can be obtained from the relation $e Z_{\mathrm{d}}=C V_{\mathrm{d}}$, where 
$C$ is the capacitance. The capacitance of an ellipsoid is expressed as $C=c_{\mathrm{f}} \varepsilon_{0}(4 \pi S)^{1 / 2}$, where $c_{\mathrm{f}}$ is a shape factor and $S$ is the surface area (Chow \& Yovanovich 1982). The shape factor for a 4:1 ellipsoid is 1.045 and the surface area of an ellipsoidal grain is $2.4 \times 10^{-9} \mathrm{~m}^{2}$, giving $Z_{\mathrm{d}}=9.4 \times 10^{4}$.

Electron capture method. Another method is based on the assumption that the dust grains have captured a substantial fraction of the free electrons so $n_{\mathrm{d}} Z_{\mathrm{d}}$ is of order $n_{\mathrm{i}}$. This gives $Z_{\mathrm{d}}=n_{\mathrm{i}} / n_{\mathrm{d}}=5 \times 10^{5}$.

\section{REFERENCES}

Baumgarten, G., \& Fricke, K. H. 2002, GeoRL, 29, 1630

Biermann, P., \& Harwit, M. 1980, ApJL, 241, L105

Chai, K.-B., \& Bellan, P. M. 2013, GeoRL, 40, 6258

Chai, K.-B., \& Bellan, P. M. 2014, JASTP, in press

Chow, Y. L., \& Yovanovich, M. M. 1982, JAP, 53, 8470

Clapp, M. L., Miller, R. E., \& Worsnop, D. R. 1995, JPhCh, 99, 6317

Davies, J. K., Roush, T. L., Cruikshank, D. P., et al. 1997, Icar, 127, 238

Du, C.-R., Thomas, H. M., Ivlev, A. V., et al. 2010, PhPl, 17, 113710

Goertz, C. K. 1989, RvGeo, 27, 271

Gupta, R., Mukai, T., Vaidya, D. B., Sen, A. K., \& Okada, Y. 2005, A\&A, 441,555

Havnes, O., Trøim, J., Blix, T., et al. 1996, JGR, 101, 10

Hayashi, C. 1981, PThPS, 70, 35

Hesstvedt, E. 1961, JGR, 66, 1985

Ilgner, M., \& Nelson, R. P. 2006, A\&A, 445, 205
Ip, W.-H. 2000, P\&SS, 48, 775

Jura, M., \& Morris, M. 1985, ApJ, 292, 482

Kahn, F. D. 1952, MNRAS, 112, 518

Lieberman, M. A., \& Lichtenberg, A. J. 2005, Principles of Plasma Discharge and Materials Processing (2nd ed.; New Jersey: Wiley)

Lucas, S., Ferry, D., Demirdjian, B., \& Suzanne, J. 2005, JPhCh, 109, 18103

Mastrapa, R. M., Sandford, S. A., Roush, T. L., Cruikshank, D. P., \& Dalle Ore, C. M. 2009, ApJ, 701, 1347

Matthews, L. S., Land, V., \& Hyde, T. W. 2012, ApJ, 744, 8

McClure, M. K., Manoj, P., Calvet, N., et al. 2012, ApJL, 759, L10

Merlino, R. L., Barkan, A., Thompson, C., \& D'Angelo, N. 1998, PhPl, 5,1607

Miyake, K., \& Nakagawa, Y. 1993, Icar, 106, 20

Oka, A., Nakamoto, T., \& Ida, S. 2011, ApJ, 738, 141

Okuzumi, S. 2009, ApJ, 698, 1122

Pollack, J. B., Hollenbach, D., Beckwith, S., et al. 1994, ApJ, 421, 615

Porco, C. C., Helfenstein, P., Thomas, P. C., et al. 2006, Sci, 311, 1393

Poulet, F., Cruikshank, D. P., Cuzzi, J. N., Roush, T. L., \& French, R. G. 2003, A\&A, 412, 305

Praburam, G., \& Goree, J. 1995, ApJ, 441, 830

Sano, T., Miyama, S. M., Umebayashi, T., \& Nakano, T. 2000, ApJ, 543,486

Seki, J., \& Hasegawa, H. 1983, Ap\&SS, 94, 177

Shimizu, S., Klumov, B., Shimizu, T., et al. 2010, JGR, 115, D18205

Stark, C. R., Potts, H. E., \& Diver, D. A. 2006, A\&A, 457, 365

Tanii, R., Itoh, Y., Kudo, T., et al. 2012, PASJ, 64, 124

Turco, R. P., Toon, O. B., Whitten, R. C., Keesee, R. G., \& Hollenbach, D. 1982, P\&SS, 3, 1147

Wardle, M. 2007, Ap\&SS, 311, 35

Zubko, V., Dwek, E., \& Arendt, R. G. 2004, ApJS, 152, 211 